\title{
Panchromatic Observations of Sn 2011dh Point to a Compact Progenitor Star
}

\section{Citation}

Soderberg, A. M., R. Margutti, B. A. Zauderer, M. Krauss, B. Katz, L. Chomiuk, J. A. Dittmann, et al. 2012. "PANCHROMATIC OBSERVATIONS OF SN 2011dh POINT TO A COMPACT PROGENITOR STAR." The Astrophysical Journal 752 (2): 78. https://doi.org/10.1088/0004-637x/752/2/78.

\section{Permanent link}

http://nrs.harvard.edu/urn-3:HUL.InstRepos:41399793

\section{Terms of Use}

This article was downloaded from Harvard University's DASH repository, and is made available under the terms and conditions applicable to Other Posted Material, as set forth at http:// nrs.harvard.edu/urn-3:HUL.InstRepos:dash.current.terms-of-use\#LAA

\section{Share Your Story}

The Harvard community has made this article openly available.

Please share how this access benefits you. Submit a story.

Accessibility 


\title{
PANCHROMATIC OBSERVATIONS OF SN 2011dh POINT TO A COMPACT PROGENITOR STAR
}

\author{
A. M. Soderberg ${ }^{1}$, R. Margutti ${ }^{1}$, B. A. Zauderer ${ }^{1}$, M. Krauss $^{2}$, B. Katz ${ }^{3,22}$, L. Chomiuk ${ }^{1,2}$, J. A. Dittmann ${ }^{1}$, E. Nakar ${ }^{4}$, \\ T. Sakamoto ${ }^{5,6}$, N. Kawai ${ }^{7}$, K. Hurley ${ }^{8}$, S. Barthelmy ${ }^{9}$, T. Toizumi ${ }^{7}$, M. Morii ${ }^{7}$, R. A. Chevalier ${ }^{10}$, M. Gurwell $^{1}$, \\ G. Petitpas ${ }^{1}$, M. Rupen ${ }^{2}$, K. D. Alexander ${ }^{1}$, E. M. Levesque ${ }^{11}$, C. Fransson ${ }^{12}$, A. Brunthaler ${ }^{13}$, M. F. Bietenholz ${ }^{14,15}$, \\ N. Chugai $^{16}$, J. Grindlay ${ }^{1}$, A. Copete ${ }^{1}$, V. Connaughton ${ }^{17}$, M. Briggs ${ }^{17}$, C. Meegan ${ }^{18}$, A. von Kienlin ${ }^{19}$, X. Zhang $^{19}$, \\ A. RAU ${ }^{19}$, S. GolenetsKiI ${ }^{20}$, E. MAZETS ${ }^{20}$, AND T. Cline ${ }^{21}$ \\ ${ }^{1}$ Harvard-Smithsonian Center for Astrophysics, 60 Garden St., Cambridge, MA 02138, USA \\ ${ }^{2}$ National Radio Astronomy Observatory, Socorro, NM 87801, USA \\ ${ }^{3}$ School of Natural Sciences, Institute for Advanced Study, Einstein Drive, Princeton, NJ 08540, USA \\ ${ }^{4}$ Raymond and Beverly Sackler School of Physics \& Astronomy, Tel Aviv University, Tel Aviv 69978, Israel \\ ${ }^{5}$ Center for Research and Exploration in Space Science and Technology (CRESST), NASA Goddard Space Flight Center, Greenbelt, MD 20771, USA \\ ${ }^{6}$ Department of Physics, University of Maryland, Baltimore County, 1000 Hilltop Circle, Baltimore, MD 21250, USA \\ ${ }^{7}$ Department of Physics, Tokyo Institute of Technology, 2-12-1 Ohokayama, Meguro, Tokyo 152-8551, Japan \\ ${ }^{8}$ Space Sciences Laboratory, University of California, 7 Gauss Way, Berkeley, CA 94720-7450, USA \\ ${ }^{9}$ NASA Goddard Space Flight Center, Greenbelt, MD 20771, USA \\ ${ }^{10}$ Astronomy Department, University of Virginia, Charlottesville, VA 22904, USA \\ ${ }^{11}$ CASA, Department of Astrophysical and Planetary Sciences, University of Colorado 389-UCB, Boulder, CO 80309, USA \\ ${ }_{12}$ Department of Astronomy, The Oskar Klein Centre, Stockholm University, 10691 Stockholm, Sweden \\ ${ }_{13}$ Max-Planck-Institute für extraterrestrische Physik, Giessenbachstraße, 85748 Garching, Germany \\ ${ }^{14}$ Department of Physics and Astronomy, York University, Toronto, M3J 1P3, Ontario, Canada \\ ${ }^{15}$ Hartebeesthoek Radio Observatory, P.O. Box 443, Krugersdorp 1740, South Africa \\ ${ }^{16}$ Institute of Astronomy, RAS, Pyatnitskaya 48, Moscow 11917, Russia \\ ${ }^{17}$ Physics Department, The University of Alabama in Huntsville, Huntsville, AL 35809, USA \\ ${ }^{18}$ Universities Space Research Association, NSSTC, 320 Sparkman Drive, Huntsville, AL 35805, USA \\ ${ }^{19}$ Max-Planck-Institut für extraterrestrische Physik, Giessenbachstrasse 1, 85748 Garching, Germany \\ ${ }^{20}$ Ioffe Physical-Technical Institute of the Russian Academy of Sciences, St. Petersburg 194021, Russia \\ ${ }^{21}$ Emeritus, NASA Goddard Space Flight Center, Code 661, Greenbelt, MD 20771, USA \\ Received 2011 July 13; accepted 2012 March 7; published 2012 May 29
}

\begin{abstract}
We report the discovery and detailed monitoring of X-ray emission associated with the Type IIb SN 2011dh using data from the Swift and Chandra satellites, placing it among the best-studied X-ray supernovae ( $\mathrm{SNe}$ ) to date. We further present millimeter and radio data obtained with the Submillimeter Array, the Combined Array for Research in Millimeter-wave Astronomy, and the Expanded Very Large Array during the first three weeks after explosion. Combining these observations with early optical photometry, we show that the panchromatic data set is well described by non-thermal synchrotron emission (radio/mm) with inverse Compton scattering (X-ray) of a thermal population of optical photons. In this scenario, the shock partition fractions deviate from equipartition by a factor, $\left(\epsilon_{e} / \epsilon_{B}\right) \sim 30$. We derive the properties of the shock wave and the circumstellar environment and find a time-averaged shock velocity of $\bar{v} \approx 0.1 c$ and a progenitor mass-loss rate of $\dot{M} \approx 6 \times 10^{-5} M_{\odot} \mathrm{yr}^{-1}$ (for an assumed wind velocity, $v_{w}=1000 \mathrm{~km} \mathrm{~s}^{-1}$ ). We show that these properties are consistent with the sub-class of Type IIb SNe characterized by compact progenitors (Type cIIb) and dissimilar from those with extended progenitors (Type eIIb). Furthermore, we consider the early optical emission in the context of a cooling envelope model to estimate a progenitor radius of $R_{*} \approx 10^{11} \mathrm{~cm}$, in line with the expectations for a Type cIIb SN. Together, these diagnostics are difficult to reconcile with the extended radius of the putative yellow supergiant progenitor star identified in archival Hubble Space Telescope observations, unless the stellar density profile is unusual. Finally, we searched for the high-energy shock breakout pulse using X-ray and gamma-ray observations obtained during the purported explosion date range. Based on the compact radius of the progenitor, we estimate that the shock breakout pulse was detectable with current instruments but likely missed due to their limited temporal/spatial coverage. Future all-sky missions will regularly detect shock breakout emission from compact SN progenitors enabling prompt follow-up observations with sensitive multi-wavelength facilities.
\end{abstract}

Key word: supernovae: individual (SN 2011dh)

Online-only material: color figures

\section{INTRODUCTION}

A key open question in the study of core-collapse supernovae $(\mathrm{SNe})$ is the nature and diversity of their progenitor systems. High-resolution optical imaging of nearby galaxies has firmly established that the progenitors of Type IIP SNe are

\footnotetext{
${ }^{22}$ Bachall Fellow, Einstein Fellow.
}

red supergiants (Smartt 2009), while pre-discovery imaging for Type II SNe 1987A (Gilmozzi et al. 1987), 1993J (Aldering et al. 1994), and 2005gl (Gal-Yam et al. 2007; Gal-Yam \& Leonard 2009) point to a diverse set of massive stars. An alternative route to the nature of the progenitors is to obtain panchromatic follow-up observations within days of explosion. The shock breakout pulse and subsequent adiabatic cooling of the ejecta can yield information on the progenitor 
size since the duration and energy of these signals scales with the size of the progenitor star (Colgate 1974; Ensman \& Burrows 1992; Waxman et al. 2007; Chevalier \& Fransson 2008; Katz et al. 2010; Nakar \& Sari 2010). Complementary follow-up observations at radio, millimeter, and X-ray bands provide unique diagnostics on the shock wave velocity which scale inversely with the progenitor radius. The utility of such a multiwavelength technique was demonstrated by the serendipitous X-ray discovery and comprehensive follow-up study of SN 2008D (Soderberg et al. 2008; Chevalier \& Fransson 2008).

On May 31.893 UT amateur astronomer Amadee Riou discovered an optical transient in M51 $(d=8.4 \pm 0.6 \mathrm{Mpc}$; Feldmeier et al. 1997; see also Vinko et al. 2012). Multiple individuals and groups subsequently confirmed the transient using pre- and post-discovery imaging (Griga et al. 2011; Silverman et al. 2011). The Palomar Transient Factory (PTF; Law et al. 2009) reported a deep non-detection in pre-discovery data constraining the onset of the optical emission to be May 31.275-31.893 UT (Arcavi et al. 2011b). Based on an initial spectrum on June 3.3 UT, the transient was classified as a Type II SN, designated SN 2011dh (Silverman et al. 2011). Further spectroscopy revealed evidence for helium absorption features prompting the re-classification as Type IIb (Arcavi et al. 2011a; Marion et al. 2011).

A putative progenitor star has been identified in pre-explosion Hubble Space Telescope (HST) images with a spectral energy distribution consistent with a yellow supergiant (Maund et al. 2011; Van Dyk et al. 2011). The mass of the star is estimated to be between $M_{\mathrm{ZAMS}} \approx 13$ and $21 M_{\odot}$, but temperaturedependent bolometric corrections, discrepancies between evolutionary tracks, and treatments of rotation should also be carefully considered (Drout et al. 2009). Based on the estimated luminosity and temperature of the object, the stellar radius is $R_{*} \approx 10^{13} \mathrm{~cm}$ (Prieto \& Hornoch 2011). Both Maund et al. (2011) and Van Dyk et al. (2011) discuss the possibility that the yellow supergiant is instead the binary companion to the SN 2011dh progenitor star. In this scenario, the actual progenitor star would have had a smaller radius.

Recently, Chevalier \& Soderberg (2010) proposed that $\mathrm{SNe}$ IIb may be divided into two sub-classes based on the radius and mass-loss history of the progenitor star and the properties of the shock wave. In this framework, compact progenitors $\left(R_{*} \sim 10^{11} \mathrm{~cm}\right)$ with modulated radio light curves and shock wave velocities of $\bar{v} \sim 0.1 \mathrm{c}$ are identified as $\mathrm{SNe} \mathrm{cIlb}$ with members including SNe 2001ig (Ryder et al. 2004), 2003bg (Soderberg et al. 2006a), and 2008ax (Roming et al. 2009). Meanwhile, extended progenitors $\left(R_{*} \sim 10^{13} \mathrm{~cm}\right)$ with smooth radio light curves and slower shock waves are identified as SNe eIIb (e.g., SN 1993J; Bartel et al. 2002; Weiler et al. 2007). The modulated radio emission points to an unusual (perhaps episodic) mass loss that may be unique to $\mathrm{SNe}$ cIIb.

SN 2011dh showed an initial peak magnitude of $M_{g} \approx$ -16.5 mag at $\Delta t \approx 1$ day since explosion before fading quickly (Arcavi et al. 2011b; Prieto et al. 2011). The SN then rebrightened at $\Delta t \approx 5$ days. The two light-curve components may be interpreted as cooling envelope emission followed by a re-brightening due to the radioactive decay of ${ }^{56} \mathrm{Ni}$. Based on a comparison of the SN 2011dh light curve with that of SN 1993J and a photospheric temperature measurement, Arcavi et al. (2011b) proposed that SN 2011 dh belongs to the Type cIlb class.

Here, we report the discovery and monitoring of X-ray emission associated with SN 2011dh and present radio- and $\mathrm{mm}$-band detections from the first few weeks after explosion.
We show that the radio and X-ray properties are consistent with those of SNe cIIb and dissimilar from those of SNe eIIb, suggesting that the progenitor was compact at the time of explosion. This is supported by our modeling of the early cooling envelope emission, which points to a progenitor radius of $R_{*} \approx 10^{11} \mathrm{~cm}$. Together, these diagnostics suggest that the putative yellow supergiant progenitor is instead a binary companion or unrelated to the SN. Finally, we present a detailed compilation of X-ray and gamma-ray observations from multiple satellites and instruments obtained during the purported explosion date range. We estimate that the shock breakout pulse was detectable with current high-energy instruments but likely missed due to their limited temporal/spatial coverage.

\section{OBSERVATIONS}

Following the optical discovery of SN 2011dh, we initiated a prompt panchromatic follow-up campaign to map the nonthermal properties of the ejecta.

\subsection{Swift/XRT Observations}

Thanks to multiple Target-of-Opportunity (ToO) requests, Swift/X-ray Telescope (XRT) promptly observed SN 2011dh on June 3.50, just $\sim 3$ days after the explosion. As initially reported in Margutti \& Soderberg (2011), we discovered a bright X-ray source $(\mathrm{S} / \mathrm{N} \sim 10)$ at coordinates, R.A.J2000 $=13^{\mathrm{h}} 30^{\mathrm{m}} 5.18$, decl. J2000 $=+47^{\circ} 10^{\prime} 11^{\prime \prime}$. 14 (uncertainty $4^{\prime \prime}$ radius, $90 \%$ confidence) at $1^{\prime \prime}$ from the optical SN position. This data analysis reported here supersedes the preliminary analysis presented in the circulars; we find a count rate of $\sim 0.015$ counts $\mathrm{s}^{-1}$ for this first epoch. We analyzed $69 \mathrm{ks}$ of archival pre-SN Swift/XRT observations and these data reveal no X-ray source at the SN position with a $3 \sigma$ upper limit of $5.6 \times 10^{-4}$ counts $\mathrm{s}^{-1}$. This fact, coupled to the spatial coincidence of the SN, strongly suggests that the new source represents the X-ray counterpart to SN 2011dh (Figure 1).

Observations of SN 2011dh with Swift continued for the next several weeks. We retrieved and analyzed the XRT data from the HEASARC archive collected in the time period June 3 to July 3 UT (total exposure time of $137 \mathrm{ks}$ ). All XRT data were analyzed with the HEASOFT (version 6.10) software package and corresponding calibration files; standard filtering and screening criteria were applied. Due to the proximity of a nearby, steady X-ray source (Figure 1$)$, we adopted a 12 pixel $\left(\sim 28^{\prime \prime}\right)$ extraction region centered on the optical position; for lower count rates $\left(<0.0025\right.$ counts $\left.\mathrm{s}^{-1}\right)$ we reduced the extraction region to a radius of 6 pixels to increase the signal-to-noise ratio $(\mathrm{S} / \mathrm{N})$ and eliminate contamination from a nearby faint source. The background was estimated from the pre-explosion Swift/XRT data to properly account for the contamination from the extended X-ray emission associated with M51. A spectrum extracted over June 3-17 UT can be modeled by an absorbed power law with photon index $\Gamma=1.5 \pm 0.2$ (90\% c.l.) assuming a Galactic foreground column density $N_{\mathrm{H}}=1.81 \times 10^{20} \mathrm{~cm}^{-2}$ (Kalberla et al. $2005)$ and no intrinsic absorption $\left(\chi^{2} /\right.$ dof $=70.7 / 73, P$-val $=$ 0.56). Alternatively, a thermal plasma spectral model with bestfitting temperature $k T=7.5_{-3.3}^{+9.7} \mathrm{keV}(90 \%$ confidence level (c.l.)) can adequately represent the data $\left(\chi^{2} /\right.$ dof $=68.8 / 73$, $P$-val $=0.62)$. Both models give an average unabsorbed flux of $F_{X} \approx 1.65 \times 10^{-13} \mathrm{erg} \mathrm{cm}^{-2} \mathrm{~s}^{-1}(0.3-8 \mathrm{keV})$ corresponding to a luminosity, $L_{X} \approx 2 \times 10^{39} \mathrm{erg} \mathrm{s}^{-1}$. 

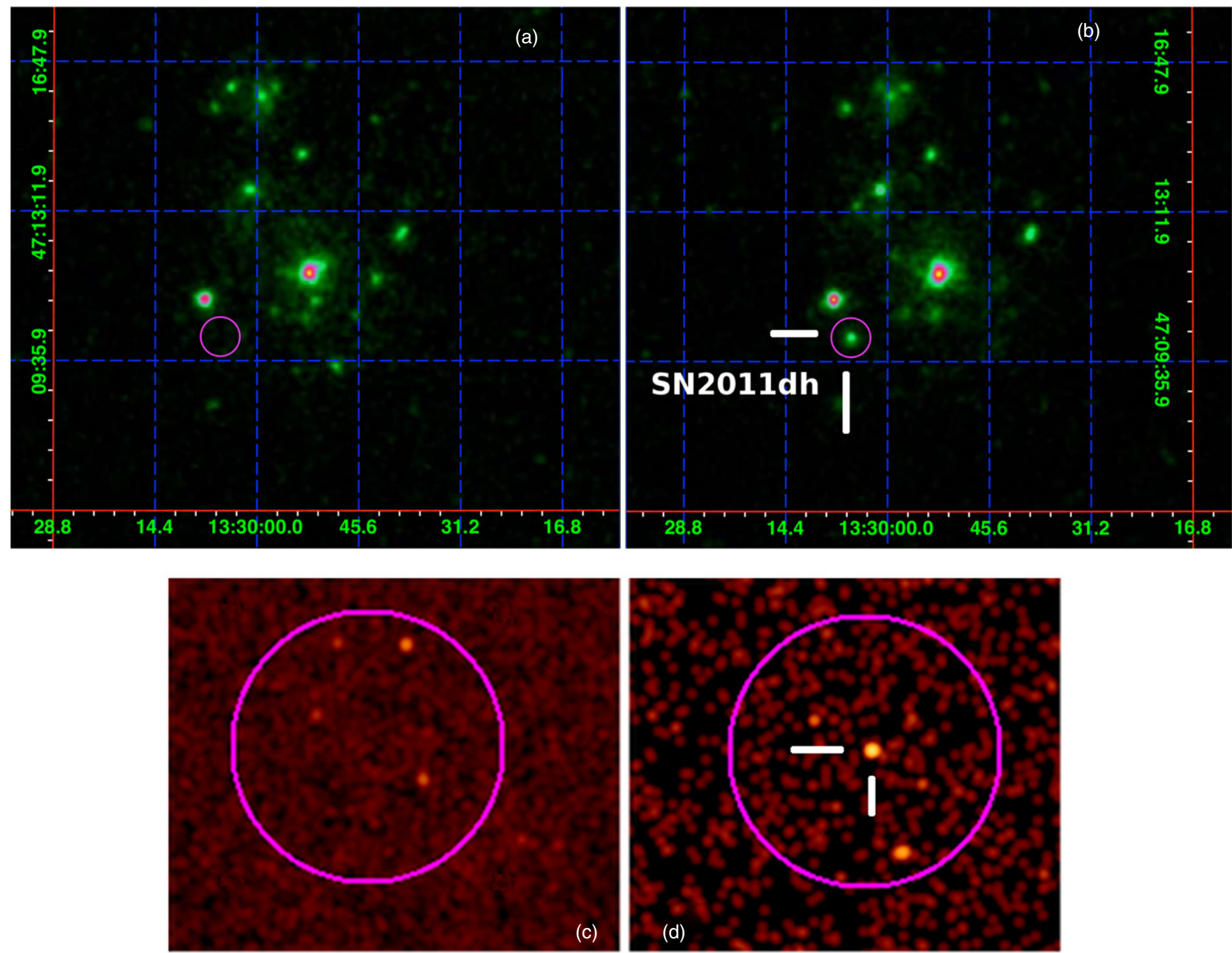

Figure 1. (a) A (0.3-10) keV co-added archival image (total exposure of $64 \mathrm{ks}$ ) of M51 obtained pre-explosion using Swift/XRT in the time range 2006 June to 2009 May. No statistically significant excess is detected at the SN2011dh position. (b) XRT observations of M51 (total exposure of 78 ks) obtained after the discovery of SN 2011dh clearly reveal an X-ray source at the SN position. (c) Archival Chandra observations reveal no source within 28 arcsec of the SN position (circle). (d) SN 2011dh is detected in our Chandra ToO observation on July 3. No bright contaminating X-ray sources are detected within the extraction region. We restrict our XRT extraction region at late times to avoid the faint source to the SW.

(A color version of this figure is available in the online journal.)

Our resulting X-ray light curve is shown in Figure 2 and Table 1 . Over the first 10 days the $\mathrm{SN}$ faded by a factor $\approx 8$. Adopting a simple power-law model for the decay, we derive an index of $\alpha=-0.8 \pm 0.2$ (90\% c.l.). The source shows some evidence for spectral softening with time, with the photon index evolving from $\Gamma_{1}=0.9 \pm 0.3$ (90\% c.l., $\Delta t=3-7$ days) to $\Gamma_{2}=1.8 \pm 0.2$ ( $90 \%$ c.l., $\Delta t=7-17$ days). In comparison with X-ray observations of SN 1993J, the softening observed for SN 2011dh begins at an earlier epoch.

\subsection{Chandra Observations}

We supplement our Swift/XRT light curve with two Chandra observations. As reported by Pooley (2011), SN 2011dh was observed with Chandra for $10 \mathrm{ks}$ beginning on June 12.3 UT. The SN was detected with a count rate of $\sim 0.0115$ counts $\mathrm{s}^{-1}$. Adopting a power-law spectral model, they derive an X-ray flux of $F_{X}=(1.0 \pm 0.3) \times 10^{-13} \mathrm{erg} \mathrm{cm}^{-2} \mathrm{~s}^{-1}(0.5-8 \mathrm{keV})$.

On July 3.4 UT, we obtained a second $10 \mathrm{ks}$ observation of SN 2011dh with the Chandra Advanced CCD Imaging Spectrometer (ACIS) under a ToO program (PI: Soderberg).
Data were reduced with the CIAO software package (version 4.3), with calibration database CALDB (version 4.4.2). We applied standard filtering using CIAO threads for ACIS data. We clearly detect a source at the SN position. Extracting within a 15 pixel aperture, we derive a (background-subtracted) count rate of $\sim 0.0049$ counts s$^{-1}$. Adopting the same power-law spectral model described in Section 2.1, we derive an unabsorbed X-ray flux of $F_{X}=(2.75 \pm 0.55) \times 10^{-14} \mathrm{erg} \mathrm{s}^{-1} \mathrm{~cm}^{-2}$ $(0.3-8 \mathrm{keV})$. We further note that we do not detect any bright nearby sources that would otherwise contaminate the extraction region adopted for the Swift/XRT data.

In Table 1 and Figure 2, we report the combined Swift/XRT and Chandra light curve of SN 2011dh, representing the bestsampled X-ray light curve for an SN IIb to date. We compare the X-ray properties of SN 2011dh with those of other SNe IIb including SNe 1993J (Chandra et al. 2009), 2001gd (PérezTorres et al. 2005), 2008ax (Roming et al. 2009), 2001ig (Schlegel \& Ryder 2002), and 2003bg (Soderberg et al. 2006a). As is clear from the figure, the SN 2011dh X-ray light curve is more closely related to Type cIIb explosions and a factor of $\sim 10$ less luminous than that observed for the Type eIIb $\mathrm{SNe}$ 


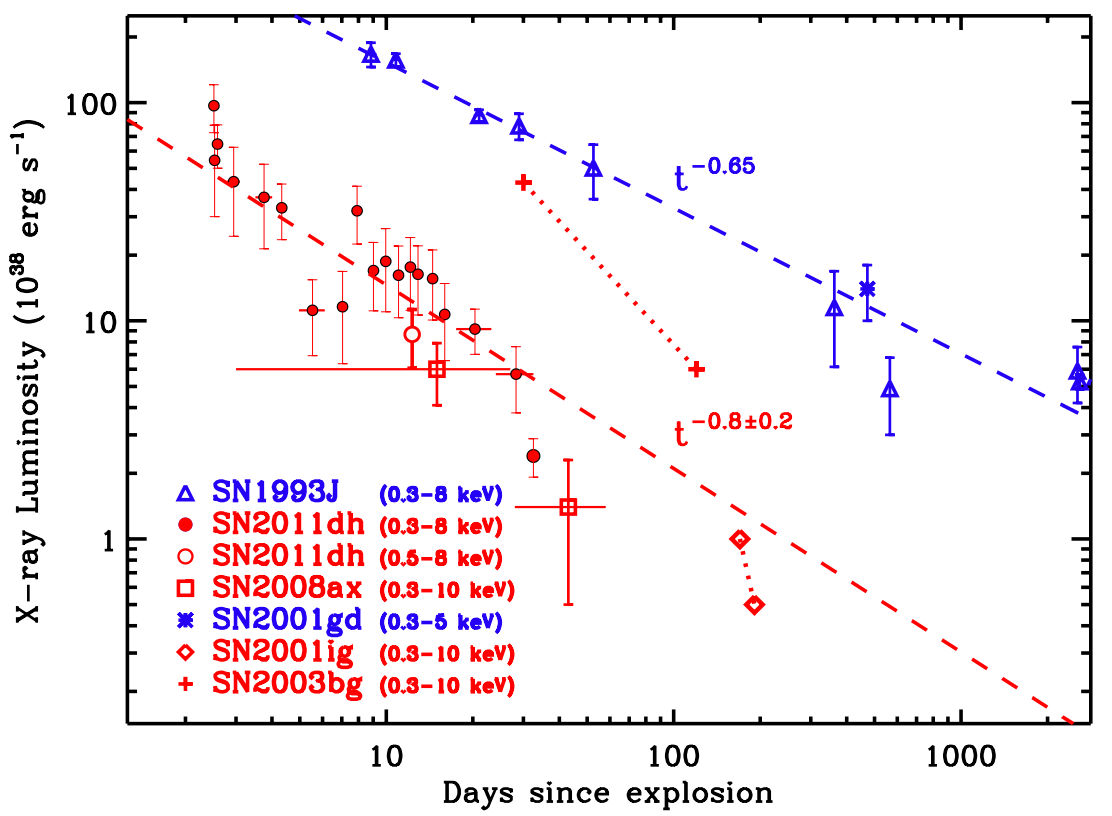

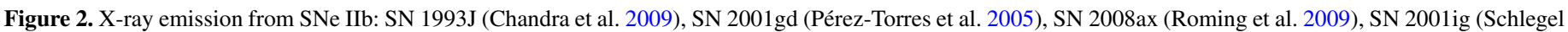

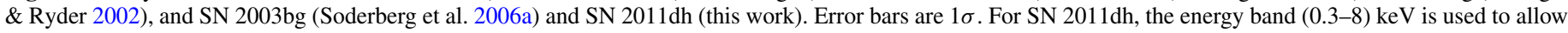

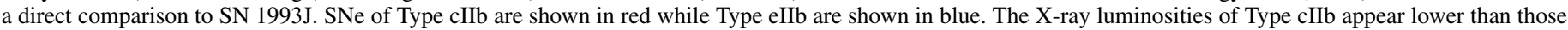
of Type eIIb.

(A color version of this figure is available in the online journal.)

Table 1

X-Ray Observations of SN 2011dh

\begin{tabular}{lcccc}
\hline \hline $\begin{array}{l}\text { Date } \\
\text { (UT) }\end{array}$ & $\begin{array}{c}\text { Time Range } \\
(\text { days })\end{array}$ & $\begin{array}{c}\text { Unabsorbed Flux } \\
\left(\mathrm{erg} \mathrm{cm}^{-2} \mathrm{~s}^{-1}\right)\end{array}$ & $\begin{array}{c}\text { Error } \\
\left(\mathrm{erg}^{-2} \mathrm{~s}^{-1}\right)\end{array}$ & Satellite \\
\hline June 3.512 & 0.004 & $1.12 \times 10^{-12}$ & $2.8 \times 10^{-13}$ & Swift/XRT \\
June 3.526 & 0.003 & $6.28 \times 10^{-13}$ & $2.8 \times 10^{-13}$ & Swift/XRT \\
June 3.585 & 0.007 & $7.45 \times 10^{-13}$ & $1.7 \times 10^{-13}$ & Swift/XRT \\
June 3.943 & 0.152 & $5.01 \times 10^{-13}$ & $2.2 \times 10^{-13}$ & Swift/XRT \\
June 4.753 & 0.252 & $4.25 \times 10^{-13}$ & $1.8 \times 10^{-13}$ & Swift/XRT \\
June 5.326 & 0.033 & $3.80 \times 10^{-13}$ & $1.1 \times 10^{-13}$ & Swift/XRT \\
June 6.536 & 0.571 & $1.29 \times 10^{-13}$ & $4.9 \times 10^{-14}$ & Swift/XRT \\
June 8.042 & 0.181 & $1.34 \times 10^{-13}$ & $6.0 \times 10^{-14}$ & Swift/XRT \\
June 8.911 & 0.253 & $3.69 \times 10^{-13}$ & $1.1 \times 10^{-13}$ & Swift/XRT \\
June 10.020 & 0.301 & $1.96 \times 10^{-13}$ & $6.8 \times 10^{-14}$ & Swift/XRT \\
June 10.954 & 0.165 & $2.16 \times 10^{-13}$ & $8.9 \times 10^{-14}$ & Swift/XRT \\
June 12.027 & 0.370 & $1.87 \times 10^{-13}$ & $6.7 \times 10^{-14}$ & Swift/XRT \\
June 13.127 & 0.179 & $2.03 \times 10^{-13}$ & $7.4 \times 10^{-14}$ & Swift/XRT \\
June 13.887 & 0.200 & $1.89 \times 10^{-13}$ & $6.6 \times 10^{-14}$ & Swift/XRT \\
June 15.493 & 0.601 & $1.80 \times 10^{-13}$ & $6.4 \times 10^{-14}$ & Swift/XRT \\
June 16.969 & 0.136 & $1.23 \times 10^{-13}$ & $4.8 \times 10^{-14}$ & Swift/XRT \\
June 21.345 & 2.848 & $1.06 \times 10^{-13}$ & $2.5 \times 10^{-14}$ & Swift/XRT \\
June 29.278 & 4.227 & $6.57 \times 10^{-14}$ & $2.2 \times 10^{-14}$ & Swift/XRT \\
\hline June 12.300 & 0.115 & $1.00 \times 10^{-13}$ & $3.0 \times 10^{-14}$ & Chandra/ACIS ${ }^{-13}$ \\
July 3.400 & 0.115 & $2.75 \times 10^{-14}$ & $5.5 \times 10^{-15}$ & Chandra/ACIS \\
\hline
\end{tabular}

Notes.

a Energy range (0.3-8) keV.

b Observation reported by Pooley (2011) within energy range (0.5-8) keV.

1993J and 2001gd. We suggest that the Type cIIb sub-class may be further characterized by low X-ray luminosities and early spectral softening.

\subsection{CARMA Observations}

We observed SN 2011dh with the Combined Array for Research in Millimeter-wave Astronomy (CARMA; Bock et al. 2006) beginning on 2011 June 4.1 UT. Observations were conducted with CARMA's nine $6.1 \mathrm{~m}$ antennas and six $10.4 \mathrm{~m}$ antennas in the $\mathrm{D}$ configuration, with a maximum baseline length of $150 \mathrm{~m}$. We implemented radio and optical pointing (Corder et al. 2010). We selected central frequencies of $v=107 \mathrm{GHz}$ and $230 \mathrm{GHz}$, with a total bandwidth of $\sim 6 \mathrm{GHz}$. Gain calibration was performed with $\mathrm{J} 1153+495$ and we used a source-calibrator cycle time of 15-20 minutes. Flux and bandpass calibration was carried out using observations of $3 \mathrm{C} 273$ and 3C345 and Neptune resulting in an overall uncertainty in the absolute flux calibration of $\sim 10 \%$. We used the Multichannel Image Reconstruction Image Analysis and Display (MIRIAD; Sault et al. 1995) software package for data reduction. We integrated on SN 2011dh for 43 minutes at each frequency; a source is clearly detected at $v=107 \mathrm{GHz}$ that is coincident with the optical and X-ray SN positions. Preliminary results for the $v=107 \mathrm{GHz}$ observation were presented by Horesh et al. (2011b). Here, we present the results of the $v=230 \mathrm{GHz}$ observation and a re-analysis of the $v=107 \mathrm{GHz}$ data in which we have refined the flux calibration. Fitting a Gaussian model to the source, we derive an integrated flux density of $F_{v}=4.5 \pm 0.3$ mJy at $v=107 \mathrm{GHz}$ and a $3 \sigma$ upper limit of $F_{v} \lesssim 3.5 \mathrm{mJy}$ at $v=230 \mathrm{GHz}$ (Table 2). Thus, the radio spectrum is optically thin between the two millimeter $(\mathrm{mm})$ bands at $\Delta t \approx 4$ days since explosion.

\subsection{SMA Observations}

Contemporaneously with the CARMA observations, we observed SN 2011dh with the Submillimeter Array (SMA; Ho et al. 2004) on June 4.0 UT in the compact configuration at a frequency of $v=230 \mathrm{GHz}$ with $8 \mathrm{GHz}$ bandwidth. Observations included all eight antennas. Passband calibration was performed in the standard way using Neptune, 3C454.3 and 3C279. We used 3C279 for flux calibration, verifying our calibration with observations of Titan in addition to 3C279 and our gain calibrators on June $10 \mathrm{UT}$. The absolute flux calibration is accurate 
Table 2

Radio and Millimeter Observations of SN 2011dh

\begin{tabular}{ccccc}
\hline \hline $\begin{array}{c}\text { Date } \\
(\mathrm{UT})\end{array}$ & $\begin{array}{c}\text { Central Frequency } \\
(\mathrm{GHz})\end{array}$ & $\begin{array}{c}\text { Flux Density } \\
(\mathrm{mJy})\end{array}$ & $\begin{array}{c}\text { Error } \\
(\mathrm{mJy})\end{array}$ & Telescope \\
\hline June 4 & 22.5 & 2.68 & 0.10 & EVLA $^{\mathrm{a}}$ \\
$\ldots$ & 107 & 4.5 & 0.3 & CARMA \\
$\ldots$ & 230 & $\leqslant 3.5$ & $\ldots$ & CARMA \\
$\ldots$ & 230 & 3.6 & 0.9 & SMA \\
\hline June 17 & 5.0 & 2.430 & 0.037 & EVLA \\
$\ldots$ & 6.8 & 4.090 & 0.048 & EVLA \\
$\ldots$ & 8.4 & 5.535 & 0.057 & EVLA \\
$\ldots$ & 13.5 & 6.805 & 0.072 & EVLA \\
$\ldots$ & 16.0 & 6.721 & 0.070 & EVLA \\
$\ldots$ & 20.5 & 6.472 & 0.195 & EVLA \\
$\ldots$ & 25.0 & 5.127 & 0.155 & EVLA \\
$\ldots$ & 29.0 & 4.603 & 0.140 & EVLA \\
$\ldots$ & 36.0 & 3.473 & 0.108 & EVLA \\
\hline
\end{tabular}

Note. ${ }^{a}$ Observation reported by Horesh et al. (2011a).

to $\sim 10 \%$. We flagged low elevation data $\left(<21^{\circ}\right)$ and the first several hours of the observation when weather conditions were less favorable, prior to improved pointing solutions, and when one antenna was missing. The data were calibrated using standard MIR/IDL routines developed for the SMA, with further calibration and imaging carried out in MIRIAD and the Astronomical Image Processing System (AIPS; Greisen 2003). The resulting total integration time on source was $2.75 \mathrm{hr}$. We detect a radio source coincident with the $\mathrm{SN}$ position with a flux density of $F_{v}=3.6 \pm 0.9 \mathrm{mJy}$ (Table 2). This detection is not inconsistent with the $3 \sigma$ upper limit from CARMA.

\subsection{EVLA Observations}

On June 4.25 UT, a radio counterpart was detected with the Expanded Very Large Array (EVLA; Perley et al. 2009) with a flux density of $F_{v}=2.68 \pm 0.10 \mathrm{mJy}$ at $v=22.5 \mathrm{GHz}$ (Horesh et al. 2011a). There is no coincident radio source in the catalog of M51 compact radio sources (Maddox et al. 2007). A comparison with the CARMA and SMA flux densities obtained contemporaneously indicates that the spectral peak lies between the EVLA and CARMA bands (Figure 3).

We began monitoring SN 2011dh with the EVLA on June 17 UT ( $\Delta t \approx 17$ days after explosion) as part of a Rapid Response observing program for long-term monitoring of the SN (PI: Soderberg). Data were collected within the wide $C, X, K u, K$, and $K a$ bands. Within each of these bands (except $X$ ), we selected two central frequencies enabling spectral coverage spanning $v=5.0-36.0 \mathrm{GHz}$. Each central frequency has associated bandwidth of $0.8-1.0 \mathrm{GHz}$. All observations were obtained in the (most extended) A-array. We used J1327+4326 to monitor the phase while the absolute flux calibration was carried out using 3C286. Data were reduced using AIPS and the Common Astronomy Software Applications. We fit a Gaussian model to the radio SN emission in each observation to derive the integrated flux density (Table 2). The reported flux density errors include errors from Gaussian fitting, the map rms noise, and systematic errors of $1 \%$ at low frequencies $(5-16 \mathrm{GHz})$ and $3 \%$ at high frequencies $(20.5-36.0 \mathrm{GHz})$. At this epoch, the peak of the radio spectrum has clearly shifted to the centimeter $(\mathrm{cm})$ band.

Additional observations with EVLA are ongoing and are the focus of a follow-up paper (Krauss et al. 2012). In Figure 3, we compare the radio spectrum of SN 2011dh with the newly

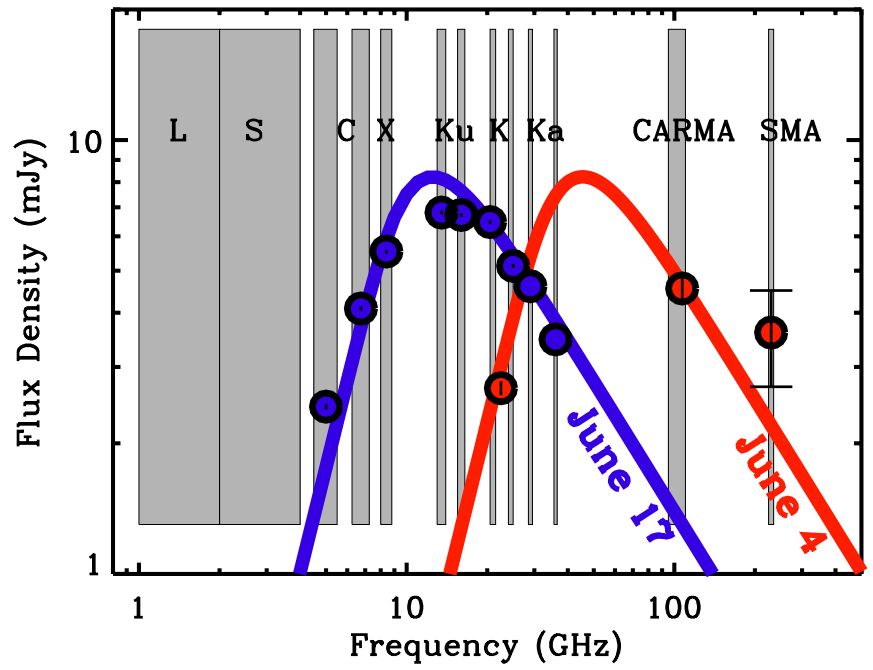

Figure 3. Radio spectrum of SN $2011 \mathrm{dh}$ across multiple epochs- $\Delta t \approx 4$ (red) and 17 (blue) days - is well described by a synchrotron self-absorbed spectral model with $F \propto v^{5 / 2}\left(F_{v} \propto v^{-(p-1) / 2}\right)$ below (above) the spectral peak, $v_{p}$. The observations indicate an electron energy index of $p \approx 3$. Error bars are $1 \sigma$. The gray bands mark the EVLA, CARMA, and SMA bands used in our long-term study of SN 2011dh as the spectral peak cascades to lower frequencies with time (see Krauss et al. 2012 for a detailed discussion).

(A color version of this figure is available in the online journal.)

available and unprecedented wide bands of EVLA which enable continuous spectral coverage from $\sim 1$ to $40 \mathrm{GHz}$. SN 2011dh represents the first $\mathrm{SN}$ for which such detailed mapping of the spectrum has been possible in the EVLA era.

\section{A MODEL FOR THE RADIO EMISSION}

Early radio observations of $\mathrm{SNe}$ uniquely trace the shock wave as it races ahead of the bulk ejecta and shock-accelerates particles in the local circumstellar medium (CSM; Chevalier 1982). This environment was enriched by the progenitor star wind during the centuries leading up to the explosion. Through this dynamical interaction, the shock wave accelerates CSM electrons into a power-law distribution, $N(\gamma) \propto \gamma^{-p}$, above a minimum Lorentz factor, $\gamma_{m}$. The accelerated electrons gyrate in amplified magnetic fields giving rise to non-thermal synchrotron emission. In the case of Type Ibc and cIIb SNe, the radio emission is quenched at low frequencies primarily due to synchrotron self-absorption (SSA), producing a spectral turnover that defines the peak of the radio spectrum, $v_{p}$. The self-absorbed radio spectrum is described by $F_{v} \propto v^{5 / 2}$ below $v_{p}$ and $F_{v} \propto v^{-(p-1) / 2}$ above $v_{p}$. As shown in Figure 3, our EVLA, CARMA, and SMA observations of SN 2011dh on two separate epochs are well described by a synchrotron self-absorbed spectrum with $p \approx 3$. We note that the modest disagreement between the SSA model and the measurements near the spectral peak may indicate asphericity of the emitting region (see Krauss et al. 2012 for a detailed discussion).

Chevalier (1998) showed that for radio $\mathrm{SNe}$ with minimal free-free absorption, the radius of the shock wave, $R$, and its time-averaged velocity, $\bar{v}$, can be robustly estimated from the observed values of $v_{p}$ and the associated peak spectral luminosity, $L_{v, p}$. As the shock wave decelerates, the optical depth to absorption processes declines and $v_{p}$ cascades to lower frequencies; for the case of free expansion, we expect $v_{p} \propto t^{-1}$ and a nearly constant peak spectral luminosity (Chevalier 1998). For $p \approx 3$, the shock wave radius is given by $R \approx 3.3 \times 10^{15}\left(\epsilon_{e} / \epsilon_{B}\right)^{-1 / 19}\left(L_{v_{p}, 26}\right)^{9 / 19} v_{p, 5}^{-1} \mathrm{~cm}$, where $L_{v_{p}, 26}$ 


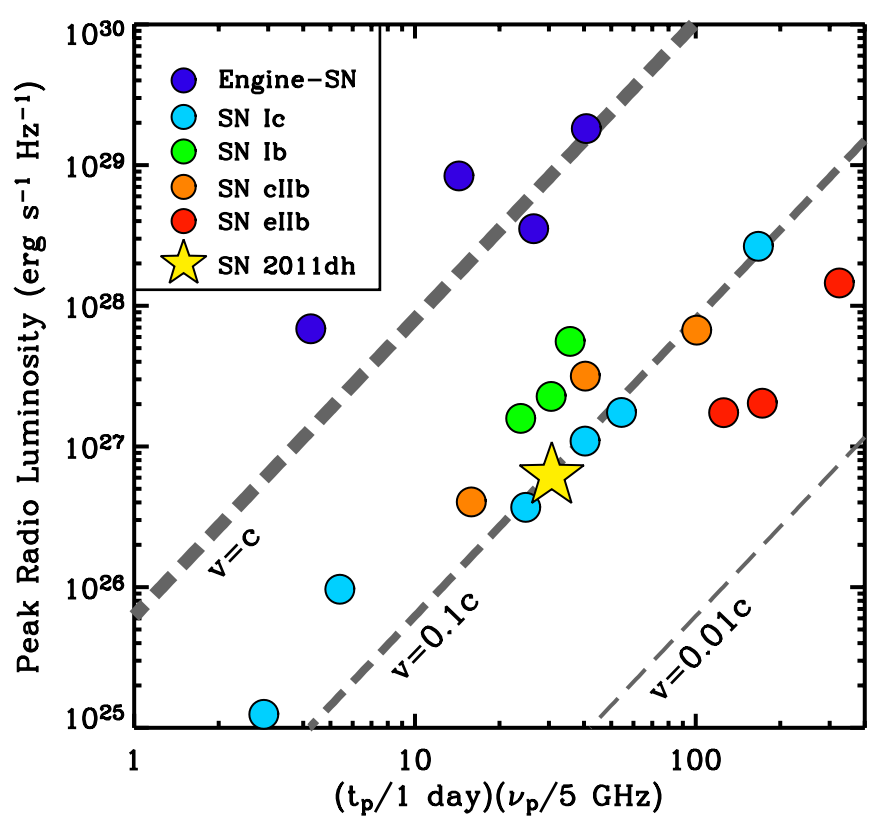

Figure 4. Radio properties of various $\mathrm{SNe}$ are compared including $\mathrm{SNe} \mathrm{Ib}$ (green), Ic (cyan), cIIb (orange), eIIb (red), and engine-driven SNe associated with nearby gamma-ray bursts (blue). The properties of the spectral peak can be used to derive the shock wave velocity (dashed lines). With a blast wave velocity of $\bar{v} \approx 0.1 c, \mathrm{SN} 2011 \mathrm{dh}$ (yellow star) is more similar to Types Ibc and cIIb than Type eIIb. Adapted from Chevalier \& Soderberg (2010).

(A color version of this figure is available in the online journal.)

is normalized to $10^{26} \mathrm{erg} \mathrm{s}^{-1} \mathrm{~Hz}^{-1}$ and $v_{p, 5}$ is normalized to $5 \mathrm{GHz}$ (Chevalier \& Fransson 2006). The fractions of post-shock energy density shared by accelerated electrons and amplified magnetic fields are denoted by $\epsilon_{e}$ and $\epsilon_{B}$, respectively, and we assume that the radio-emitting region is half of the total volume enclosed by a spherical shock wave.

As shown in Figure 3, for SN 2011dh we find $v_{p} \approx 40$ and $\approx 11 \mathrm{GHz}$ on 2011 June 4 and 17 ( $\Delta t \approx 4$ and 17 days), respectively, with an associated peak luminosity of $L_{v_{p}, 26} \approx 6.7$ on both epochs. These observables correspond to shock wave radii of $R \approx(1.0,3.7) \times 10^{15} \mathrm{~cm}$ assuming typical partition values of $\epsilon_{e}=\epsilon_{B}=0.1$. The time-averaged shock wave velocity is thus $R / \Delta t \approx \bar{v} \approx 0.1 c$ and thus a factor of $\sim 2$ faster than the material at the optical photosphere at $\Delta t \approx 3$ days (Silverman et al. 2011). The total internal energy required to power the observed radio signal can be estimated from the post-shock magnetic energy density, $E=B^{2} R^{3} / 12 \epsilon_{B}$. As shown by Chevalier \& Fransson (2006), the amplified magnetic field is directly determined from the spectral properties, $B \approx$ $0.70\left(\epsilon_{e} / \epsilon_{B}\right)^{-4 / 19} L_{v_{p}, 26}^{-2 / 19} v_{p, 5}$ G. At $\Delta t \approx 4$ and 17 days, we find $B \approx 4.5$ and $1.2 \mathrm{G}$. The total internal energy is thus $E \approx$ $(1.7,6.3) \times 10^{46} \mathrm{erg}$ for the two epochs, respectively, by maintaining the assumption that $\epsilon_{B}=0.1$, i.e., $\epsilon_{B,-1}$. The roughly linear temporal increase of internal energy is consistent with a slightly decelerated shock wave. As shown in Figure 4, the shock wave properties of SN 2011dh are similar to those of Type cIIb, which tend to also be characterized by shock wave velocities of $\bar{v} \gtrsim 0.1 c$ while slower shock waves are inferred for $\mathrm{SNe}$ eIIb.

The progenitor mass-loss rate is $\dot{M} \approx 0.39 \times 10^{-5} \epsilon_{B,-1}^{-1}$ $\left(\epsilon_{e} / \epsilon_{B}\right)^{-8 / 19} L_{v_{p}, 26}^{-4 / 19} v_{p, 5}^{2} t_{p, 10}^{2} M_{\odot} \mathrm{yr}^{-1}$, where $t_{p, 10}$ is the observed time of the spectral peak normalized to 10 days and we have assumed a wind velocity of $v_{w}=1000 \mathrm{~km} \mathrm{~s}^{-1}$. Thus, we estimate a progenitor mass-loss rate for SN 2011dh of $\dot{M} \approx 3 \times 10^{-5} M_{\odot} \mathrm{yr}^{-1}$, similar to the mass-loss rates derived for SNe Ibc and cIIb and also similar to the values observed for Galactic Wolf-Rayet stars (Cappa et al. 2004; Crowther 2007), and a factor of 100 lower than the mass-loss rate inferred for SN 1993J (Fransson et al. 1996). We note that in this framework, the radio data constrain the ratio, $\left(\dot{M} / v_{w}\right)$, such that a variation in the assumed value of $v_{w}$ shifts the mass-loss rate estimate by the same factor.

\section{A MODEL FOR THE X-RAY EMISSION}

On timescales of days after explosion, the X-ray emission observed from SNe Ibc and IIb may be dominated by a number of different emission processes including synchrotron, thermal, and inverse Compton (IC) scattering. Continued energy input from a compact remnant (black hole, magnetar) has also been invoked to explain the X-ray emission from some events (e.g., SN 2006aj, Mazzali et al. 2006; Soderberg et al. 2006b; SN 1979C, Patnaude et al. 2011). The temporal and spectral evolution of the radio and X-ray emission, together with their observed luminosity values, enables these different processes to be distinguished. Here, we consider the nature of the observed X-ray light curve for SN 2011dh.

The radio-to-X-ray spectral index is observed to be $\beta_{R X} \approx$ -0.7 on both June 4 and 17 UT. Thus, an extrapolation of the optically thin radio synchrotron spectrum with $p \approx 3$ underestimates the X-ray flux at both epochs by a factor of $\sim$ 140. Similarly high radio-to-X-ray spectral indices are not atypical for SNe Ibc and IIb (see Chevalier \& Fransson 2006 and references within). Attributing the X-rays to synchrotron emission would require efficient particle acceleration and a flattening of the electron distribution at high energies.

As shown in Chevalier \& Fransson (2003), the thermal emission from shock-heated ejecta in the reverse shock region may give rise to strong free-free X-ray emission. If the material is fully ionized and in temperature equilibrium, the expected luminosity is broadly determined by the electron temperature and the mass-loss rate of the progenitor star with an expected linear decay in time. We use Equations (25)-(30) of Chevalier $\&$ Fransson (2006) with $\bar{v} \approx 0.1 c$ and $\dot{M} \approx 3 \times 10^{-5} M_{\odot} \mathrm{yr}^{-1}$ to estimate the electron temperature. ${ }^{23}$ The expected free-free X-ray luminosity for SN $2011 \mathrm{dh}$ is then $L_{\mathrm{ff}} \approx 2.5 \times 10^{37} \mathrm{erg} \mathrm{s}^{-1}$ on June 4 UT. This is a factor of 200 lower than the Swift/XRT measurement at this epoch (Figure 2).

We next consider an IC scattering model in which the optical photons associated with both envelope cooling emission and the radioactive decay of ${ }^{56} \mathrm{Ni}$ are upscattered to the X-ray band by radio-emitting electrons (Chevalier et al. 2006). In this scenario, the X-ray decay should track the optical evolution through the cooling envelope decay to the re-brightening due to ${ }^{56} \mathrm{Ni}$ decay. The early optical emission indicates an average luminosity of $L_{\mathrm{bol}} \sim$ few $\times 10^{42} \mathrm{erg} \mathrm{s}^{-1}$ and a minimum near $\Delta t \approx 5$ days. The $\mathrm{X}$-ray light curve suggests a similar minimum near this epoch and an overall decay of roughly $L_{X} \propto t^{-1}$ (Figure 2). Adopting the formalism of Chevalier \& Fransson (2006) and assuming the relativistic electron population extends down to $\left(\gamma_{m}=1\right)$, the predicted IC emission is $L_{\mathrm{IC}} \approx$ $2.9 \times 10^{36} \epsilon_{B,-1} \dot{M}_{-5}\left(\epsilon_{e} / \epsilon_{B}\right)^{11 / 19}(v / 0.1 c)^{-1} L_{\mathrm{bol}, 42} \Delta t_{d, 10}^{-1} \mathrm{erg} \mathrm{s}^{-1}$, where $\dot{M}_{-5}$ is the mass-loss rate normalized to $10^{-5} M_{\odot} \mathrm{yr}^{-1}$

${ }^{23}$ We note that Chevalier \& Fransson (2006) adopt the notation $A_{*} \equiv A /\left(5 \times 10^{11} \mathrm{~g} \mathrm{~cm}^{-1}\right)$, where $A=\dot{M} / 4 \pi v_{w}$. 
and we maintain the assumption of $v_{w}=10^{3} \mathrm{~km} \mathrm{~s}^{-1}$. Here, $\Delta t_{d, 10}$ is the time since explosion normalized to 10 days.

We find that the X-ray emission may be attributed to IC emission if the assumption of equipartition is relaxed and $\left(\epsilon_{e} / \epsilon_{B}\right) \approx 30$ with $\epsilon_{B} \approx 0.01$. This deviation from equipartition implies modest adjustments to our physical parameter estimates, including a factor of $\sim 2$ increase in the mass-loss rate and a minimal increase in the inferred total internal energy of the radio-emitting material. The modified values for the massloss rate and magnetic field are $M_{-5} \approx 6$ and $B \approx 2.2 \mathrm{G}$, respectively. A prediction of this model is that the $\mathrm{X}$-ray light curve will decay more steeply following the optical SN peak (Chevalier \& Fransson 2006), which may be suggested by our Chandra measurement on July 3 UT.

\section{CONSTRAINTS ON THE PROGENITOR SIZE}

The early optical emission from SNe is dominated by the adiabatic cooling of envelope material following the breakout of the shock wave through the stellar surface (Ensman \& Burrows 1992). This component cascades through the optical band in the first few days following explosion. The radius and temperature associated with this component may be roughly approximated as thermal (although see Nakar \& Sari 2010 for a more comprehensive discussion of the spectral evolution) and used together with estimates for the bulk SN parameters (including the ejecta kinetic energy, $E_{K}$, and mass, $M_{\mathrm{ej}}$ ) leads to a determination of the progenitor radius, $R_{*}$. Such techniques have been used to derive the progenitor radius of SNe 1987A (Ensman \& Burrows 1992), 1999ex and 2008ax (Chevalier \& Soderberg 2010), 2008D (Chevalier \& Fransson 2008; Soderberg et al. 2008), in addition to SN 2006aj associated with XRF 060218 (Campana et al. 2006; Waxman et al. 2007). Here, we adopt the formalism of Chevalier \& Fransson (2008) in which the temperature of the photosphere is given by $T_{\mathrm{ph}} \approx 7800 E_{K, 51}^{0.03} M_{\mathrm{ej}, \odot}^{-0.04} R_{*, 11}^{0.25} \Delta t_{d}^{-0.48} \mathrm{~K}$ and the photospheric radius is $R_{\mathrm{ph}} \approx 3 \times 10^{14} E_{K, 51}^{0.39} M_{\mathrm{ej}, \odot}^{-0.28} \Delta t_{d}^{0.78} \mathrm{~cm}$. Here we have normalized $E_{K, 51}$ to $10^{51} \mathrm{erg}, M_{\mathrm{ej}, \odot}$ to $M_{\odot}$, and $R_{*, 11}$ to $10^{11} \mathrm{~cm}$. Within this framework, it is assumed that the density and pressure profiles of the envelope are consistent with those of Matzner \& McKee (1999), and that the bolometric luminosity decays as $L_{\mathrm{ph}} \propto \Delta t_{d}^{-0.34}$.

We model the first few optical observations ( $\Delta t \lesssim 5$ days) including the initial detection of $L_{\mathrm{ph}} \approx 10^{42} \mathrm{erg} \mathrm{s}^{-\tau}$ on June 1.191 UT (Arcavi et al. 2011b). Silverman et al. (2011) report a photospheric velocity of $v_{\mathrm{ph}} \approx 17,600 \mathrm{~km} \mathrm{~s}^{-1}$ at $\Delta t \approx$ 3 days after explosion implying a ratio of $\left(E_{K, 51} / M_{\mathrm{ej}, \odot}\right) \sim$ few. We estimate $R_{\mathrm{ph}} \approx 5 \times 10^{14} \mathrm{~cm}$ and $T_{\mathrm{ph}} \approx 8000 \mathrm{~K}$ at $\Delta t \approx 1$ day. This is roughly consistent with the photospheric temperature derived from optical spectroscopy (Arcavi et al. $2011 \mathrm{~b})$ and implies a compact progenitor since $T_{\mathrm{ph}} \propto R_{*, 11}^{0.25}$. The high luminosity of the initial detection requires a ratio, $E_{K} / M_{\mathrm{ej}} \sim$ few, in line with the ratio implied by the high photospheric velocity. Thus, the early optical emission points to a compact progenitor, similar to those of SNe Ibc and cIIb, and consistent with the earlier reported by Arcavi et al. (2011b) and the conclusions of Murphy et al. (2011) based on a study of the SN 2011dh environment.

We note, however, that the observed optical decay, $L \propto \Delta t_{d}^{-1}$, is significantly steeper than the model prediction. This may point to an irregular density profile near the stellar surface, perhaps associated with an unusual mass-loss ejection in the final stage of the progenitor's evolution. Such an irregular density profile

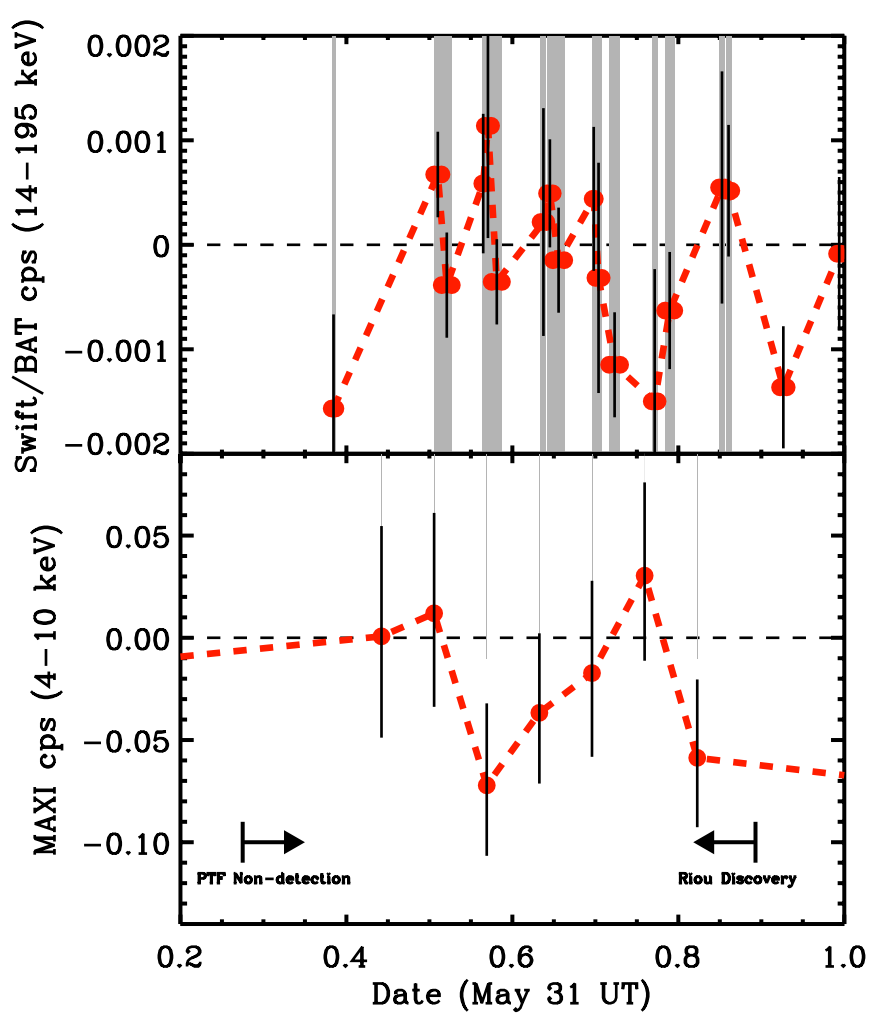

Figure 5. Swift/BAT (15-195 keV) and MAXI (4-10 keV) observations of the SN 2011dh field during the estimated explosion date range bounded by the discovery by Riou and pre-discovery limit (PTF) marked with arrows, May 31.275-31.893 UT (Arcavi et al. 2011b). No source is detected at the position of the SN during the individual $(\sim 1000 \mathrm{~s})$ BAT pointings or $(\sim 80 \mathrm{~s})$ MAXI scans. The time ranges when the SN was in the field of view of the instruments are shown in gray.

(A color version of this figure is available in the online journal.)

may affect some of the conclusions that we derived above based on the canonical model and this will be the focus of a future publication.

\section{SHOCK BREAKOUT X-RAY EMISSION}

For compact progenitors such as SN 2011dh, the breakout pulse may be detectable at X-ray and gamma-ray energies (e.g., SN 2008D; Soderberg et al. 2008). To this end, we searched for evidence of a high-energy pulse associated with the shock breakout of SN 2011dh using data collected by the Swift Burst Alert Telescope (BAT), the Monitor of All-sky X-ray Image (MAXI) camera attached to the Japanese Experiment Module, and the Interplanetary Network (IPN: Mars Odyssey, KonusWind, RHESSI, INTEGRAL (SPectrometer on INTEGRALAdvanced Camera for Surveys), Swift/BAT, Suzaku, AGILE, and Fermi/Gamma-ray Burst Monitor (GBM); we note that Messenger was in superior conjunction and off during this period).

The position of SN 2011dh was observed by Swift/BAT over many pointings during the estimated explosion date range, May 31.275-31.893 UT. No gamma-ray emission was detected from the SN. We compile the Swift/BAT observations in Figure 5. Each pointing had a duration of $\sim 500-1000 \mathrm{~s}$, and the total time on source was $11,148 \mathrm{~s}$ or $20 \%$ of the full explosion date range. We estimate the count rate at the $\mathrm{SN}$ position and also in the background in each pointing and note that the sensitivity varies as a function of the off-axis angle, ranging from 10 to $57 \mathrm{deg}$. Assuming a power-law spectrum for the breakout flux 
with a photon index of $\Gamma=2$, we infer $5 \sigma$ upper limits on the gamma-ray emission from the $\mathrm{SN}$ of $F_{\gamma} \lesssim(1.1-3.9) \times$ $10^{-9} \mathrm{erg} \mathrm{cm}^{-2} \mathrm{~s}^{-1}(15-195 \mathrm{keV})$ for each individual pointing. The combined upper limit is $F_{\gamma} \approx 2.8 \times 10^{-10} \mathrm{erg} \mathrm{cm}^{-2} \mathrm{~s}^{-1}$ for the same energy range. At the distance of M51, this limit corresponds to a luminosity of $L_{\gamma} \lesssim 2.4 \times 10^{42} \mathrm{erg} \mathrm{s}^{-1}$. We further note that no emission was detected by Swift/BAT in the direction of the SN during spacecraft slews throughout this time period; the BAT Slew Survey (Grindlay 2007) places a $\sim 4 \sigma$ upper limit within energy range (15-50) $\mathrm{keV}$.

MAXI (Matsuoka et al. 2009) is an X-ray all-sky monitor that scans most of the sky every 92 minutes with its Gas Slit Cameras (Mihara et al. 2011). Unfortunately, during the estimated period of the SN breakout, the direction of M51 was covered only by a camera with high background, resulting in less stringent limits than usual. The X-ray image taken at every scan transit $(\sim 80 \mathrm{~s})$, encoded in one dimension by the detector position and the other by transit timing, was fitted to the point-spread function with the fixed source position to evaluate the source count rate. The energy range of (4-10) keV was chosen to maximize the $\mathrm{S} / \mathrm{N}$. Then the count rate was converted to energy flux assuming a power-law model with a photon index of $\Gamma=2$ resulting in a conversion factor of $F_{X} \approx$ $1.1 \times 10^{-8} \mathrm{erg} \mathrm{cm}^{-2} \mathrm{~s}^{-1}$ (counts s$\left.{ }^{-1}\right)^{-1}$. We derive an average $5 \sigma$ upper limit of $L_{X} \lesssim 3.9 \times 10^{42} \mathrm{erg} \mathrm{s}^{-1}$. In comparison, the observed breakout pulse from SN $2008 \mathrm{D}(d \approx 30 \mathrm{Mpc})$ had a luminosity $\sim 25$ times higher $\left(L_{X} \approx 10^{44} \mathrm{erg} \mathrm{s}^{-1} ; 0.3-10 \mathrm{keV}\right)$ with a duration of $\sim 5$ minutes.

While Swift/BAT and MAXI both have a limited temporal coverage, the IPN is full sky with temporal duty cycle of nearby $100 \%$; it is sensitive to hard X-ray emission in the energy range (25-150) keV (Hurley et al. 2010). Within a twoday window centered on the estimated explosion date, a total of four triggered bursts were detected by the IPN. All four were confirmed through observations by multiple instruments or spacecrafts and thus could be localized. These detections include Fermi/GBM (three bursts), Konus (one), INTEGRAL (one), and Swift/BAT (one). In all cases the localizations were statistically inconsistent at a $\gtrsim 3 \sigma$ level with the position of SN 2011dh. Thus, we find no evidence for a detected gammaray transient in coincidence with SN 2011dh and adopt an upper limit of $F_{\gamma} \lesssim 6 \times 10^{-7} \mathrm{erg} \mathrm{cm}^{-2} \mathrm{~s}^{-1}$ corresponding to an energy of $E_{\gamma} \lesssim 5 \times 10^{45} \mathrm{erg}$ for SN 2011dh.

\subsection{A Comparison to Breakout Predictions}

Here, we compare theoretical predictions for a prompt breakout pulse with the available X-ray and $\gamma$-ray limits. We consider a model in which the progenitor is compact, $R_{*, 11} \approx 1$, and embedded in a stellar wind with $\dot{M}_{-5} \approx 6$. In the standard SN Ibc model of Chevalier \& Fransson (2006), also applicable to $\mathrm{SNe}$ cIIb, the shock wave radius evolves as $R \propto t^{m}$ with $m \approx 0.9$. Extrapolating back to the breakout radius, the shock wave speed at the stellar surface is $v_{0} \approx 0.3 c$ and we define $\beta_{0} \approx 0.3 \beta_{-0.5}$ as the shock velocity (divided by $c$ ) at this time. In the case of such fast shock velocities, there are deviations from thermal equilibrium resulting in high-energy emission (Katz et al. 2010; Nakar \& Sari 2010; Sapir et al. 2011).

The optical depth at the stellar surface is $\tau=\kappa \dot{M} / 4 \pi v_{w} r \approx$ $2 \dot{M}_{-5} R_{11}^{-1}$, where we adopt $\kappa=0.4 \mathrm{~cm}^{2} \mathrm{~g}^{-1}$ and $R_{11}$ is the shock radius normalized to $10^{11} \mathrm{~cm}$. The shock breakout will occur outside of the star, within the stellar wind at a radius, $R_{\mathrm{br}}=$ $\beta_{0} \kappa \dot{M} / 4 \pi v_{w} \approx 6 \times 10^{10} \dot{M}_{-5} \beta_{-0.5} \mathrm{~cm}$ as long as $R_{*}<R_{\mathrm{br}}$.
In the case of SN 2011dh, we therefore predict a wind breakout at radius, $R_{\mathrm{br}} \approx 4 \times 10^{11} \mathrm{~cm}$. The energy associated with breakout is $E_{\mathrm{br}} \approx 3 \times 10^{43} \dot{M}_{-5}^{2} \beta_{-0.5}^{3}$ (Katz et al. 2012). On the breakout timescale, a radiative collisionless shock forms and an additional comparable or larger amount of energy is emitted (Katz et al. 2012). Adopting our parameters for SN 2011dh, we predict $E_{\mathrm{br}} \gtrsim 10^{45} \mathrm{erg}$. During the transition from a radiation-mediated shock to collisionless, the temperature rises steadily from $\gtrsim$ $\mathrm{keV}$ to $\gtrsim 100 \mathrm{keV}$ and we roughly estimate the spectrum to be $\nu F_{v} \sim$ constant.

The predicted breakout energy is within the detectability range of the Swift/BAT and MAXI upper limits spanning (4-195) $\mathrm{keV}$, however, both offer only limited temporal coverage. We estimate the rise time of the breakout pulse to be $t_{\mathrm{br}} \approx R_{\mathrm{br}} / v_{0} \approx 1$ minute. Efficient emission from the collisionless shock continues beyond this time, and the full duration of the pulse is necessarily longer. Given the large gaps in the Swift/BAT and MAXI coverage (see Figure 5), especially toward the beginning of the explosion date range, we speculate that Swift and MAXI missed the breakout pulse. The IPN upper limit is a factor of a few above the predicted energy and thus consistent with this breakout model. Finally, we note that if the progenitor was more extended with a radius, $R_{*} \approx 10^{13} \mathrm{~cm}$, a stronger pulse of $E_{\mathrm{br}} \sim 3 \times 10^{47} R_{*, 13}^{2}$ erg is expected at lower frequencies, $h v \lesssim 300 \mathrm{eV}$. Such a pulse is not constrained by the hard X-ray/gamma-ray observations presented here.

\section{CONCLUSIONS}

We present multi-wavelength follow-up observations of SN 2011dh spanning the radio, millimeter, X-ray, and gammaray bands and obtained within the first few seconds to weeks following the explosion. The X-ray light curve for SN 2011dh is perhaps the best-sampled to date for an $\mathrm{SN}$ IIb and suggests that X-ray properties (luminosity, spectral evolution) may further distinguish compact and extended progenitors. Using the newly available wide bands of the EVLA, we show that the radio and millimeter data are consistent with a synchrotron selfabsorbed spectrum, while the X-ray emission may be understood as IC upscattering of optical SN photons assuming a nonnegligible deviation from equipartition of the shock partition fractions. These data offer unique diagnostics (shock wave velocity, $\bar{v} \approx 0.1 c$ ) and point to a compact progenitor star. Through modeling of the early optical emission, we find that the photosphere is characterized by a low temperature, $T_{\mathrm{ph}} \approx 8000 \mathrm{~K}$, and the physical parameters of the ejecta are constrained to be $E_{K, 51} / M_{\mathrm{ej}, \odot} \sim$ few. These properties point to a compact progenitor, $R_{*} \approx 10^{11} \mathrm{~cm}$, however, we find that the fast decay of the early optical emission at $\Delta t \lesssim 5$ day is incompatible with canonical cooling envelope models and may suggest an irregular ejecta profile. A compact progenitor size appears inconsistent with the extended radius $\left(R_{*} \sim 10^{13} \mathrm{~cm}\right)$ of the coincident yellow supergiant identified in pre-explosion HST imaging, suggesting an unusual density profile for the outer layers of the progenitor star. It may be possible to explain the coincident object as a binary companion, however, it is estimated that the yellow supergiant phase lasts just $\sim 3000$ years (Drout et al. 2009) making this scenario improbable. At the same time, the rarity of yellow supergiants makes a positional coincidence unlikely, suggesting the supergiant is indeed related to the progenitor system.

If the supergiant was characterized by a low-mass envelope and a low-density stellar wind, it may be possible to reconcile 
the radio and $\mathrm{X}$-ray observations with the putative progenitor. Georgy (2012) recently suggested that the mass-loss rates for yellow supergiants have been theoretically overestimated. However, the early cooling envelope emission requires a substantially lower density envelope then those associated with supergiants; in this scenario, ejecta asymmetries may be the only way to reconcile the early optical data with the coincident supergiant as the progenitor.

We conclude that SN 2011dh is likely a member of the Type cIIb class of core-collapse explosions. Our long-term EVLA monitoring observations will reveal if the radio light curves are modulated (see Krauss et al. 2012 for details), indicative of a variable and/or episodic mass-loss history in the decades leading up to explosion-an observational characteristic shared by other SNe cIIb. In addition, our long-term monitoring with the Very Long Baseline Interferometer will provide a direct measurement of the shock radius and, in turn, an independent constraint on the shock partition fractions (MartiVidal et al. 2011; Bietenholz et al. 2012). Future observations of the explosion site with HST imaging will reveal whether the supergiant has disappeared, thereby directly linking it to the explosion.

Finally, we used the parameters derived above for the shock wave and progenitor to estimate that the shock breakout pulse from SN 2011dh was detectable in the X-ray and gamma-ray bands with current satellites. We attribute the lack of a detection as likely due to limited temporal/spatial coverage during the estimated explosion date. Looking forward, sensitive and wide-field X-ray experiments such as LOBSTER and JANUS will regularly discover shock breakout emission from similarly nearby and compact SN progenitors (see, e.g., Soderberg et al. 2009 for a discussion). Such detections will not only provide information on the progenitor size since the explosion date estimates will enable statistically significant searches for gravitational wave and neutrino counterparts (Ott 2009; Katz et al. 2012). Furthermore, these prompt discoveries will enable rapid low-frequency follow-up with sensitive radio and millimeter facilities thanks to the advent of EVLA, the Atacama Large Millimeter Array, ASKAP, and MeerKAT.

We thank Philip Massey, Edo Berger, Ryan Foley, Maria Drout, and Robert Kirshner for useful conversations. K.H. is grateful for IPN support from NASA grants NNX10AI23G, NNX09AU03G, NNX07AR71G, and NNX10AR12G. B.K. and E.M.L. are supported by NASA through Einstein Postdoctoral Fellowship awarded by the Chandra X-ray Center, which is operated by the Smithsonian Astrophysical Observatory for NASA under contract NAS8-03060. Support for CARMA construction was derived from the Gordon and Betty Moore Foundation, the Kenneth T. and Eileen L. Norris Foundation, the James S. McDonnell Foundation, the Associates of the California Institute of Technology, the University of Chicago, the states of California, Illinois, and Maryland, and the National Science Foundation. Ongoing CARMA development and operations are supported by the National Science Foundation under a cooperative agreement, and by the CARMA partner universities. The SMA is a joint project between the Smithsonian Astrophysical Observatory and the Academia Sinica Institute of Astronomy and Astrophysics, and is funded by the Smithsonian Institution and the Academia Sinica. The EVLA is operated by the National Radio Astronomy Observatory, a facility of the National Science Foundation operated under cooperative agreement by Associated Universities, Inc.

\section{REFERENCES}

Aldering, G., Humphreys, R. M., \& Richmond, M. 1994, AJ, 107, 662

Arcavi, I., Gal-Yam, A., Polishook, D., et al. 2011a, ATel, 3413, 1

Arcavi, I., Gal-Yam, A., Yaron, O., et al. 2011b, ApJ, 742, L18

Bartel, N., Bietenholz, M. F., Rupen, M. P., et al. 2002, ApJ, 581, 404

Beitenholz, M. F., Brunthaler, A., Soderberg, A. M., et al. 2012, ApJ, in press (arXiv:1201.0771)

Bock, D. C.-J., Bolatto, A. D., Hawkins, D. W., et al. 2006, Proc. SPIE, 6267,626713

Campana, S., Mangano, V., Blustin, A. J., et al. 2006, Nature, 442, 1008

Cappa, C., Goss, W. M., \& van der Hucht, K. A. 2004, AJ, 127, 2885

Chandra, P., Dwarkadas, V. V., Ray, A., Immler, S., \& Pooley, D. 2009, ApJ, 699, 388

Chevalier, R. A. 1982, ApJ, 258, 790

Chevalier, R. A. 1998, ApJ, 499, 810

Chevalier, R. A., \& Fransson, C. 2003, in Supernovae and Gamma-Ray Bursters, ed. K. Weiler (Lecture Notes in Physics, Vol. 598; Berlin: Springer), 171

Chevalier, R. A., \& Fransson, C. 2006, ApJ, 651, 381

Chevalier, R. A., \& Fransson, C. 2008, ApJ, 683, L135

Chevalier, R. A., Fransson, C., \& Nymark, T. K. 2006, ApJ, 641, 1029

Chevalier, R. A., \& Soderberg, A. M. 2010, ApJ, 711, L40

Colgate, S. A. 1974, ApJ, 187, 333

Corder, S. A., Wright, M. C. H., \& Carpenter, J. M. 2010, Proc. SPIE, 7733 , 77333P

Crowther, P. A. 2007, ARA\&A, 45, 177

Drout, M. R., Massey, P., Meynet, G., Tokarz, S., \& Caldwell, N. 2009, ApJ, 703, 441

Ensman, L., \& Burrows, A. 1992, ApJ, 393, 742

Feldmeier, J. J., Ciardullo, R., \& Jacoby, G. H. 1997, ApJ, 479, 231

Fransson, C., Lundqvist, P., \& Chevalier, R. A. 1996, ApJ, 461, 993

Gal-Yam, A., \& Leonard, D. C. 2009, Nature, 458, 865

Gal-Yam, A., Leonard, D. C., Fox, D. B., et al. 2007, ApJ, 656, 372

Georgy, C. 2012, A\&A, 538, L8

Gilmozzi, R., Cassatella, A., Clavel, J., et al. 1987, Nature, 328, 318

Greisen, E. W. 2003, in Information Handling in Astronomy - Historical Vistas, ed. A. Heck (Astrophysics and Space Science Library, Vol. 285; Dordrecht: Kluwer), 109

Griga, T., Marulla, A., Grenier, A., et al. 2011, CBET, 2736, 1

Grindlay, J. E. 2007, in ASP Conf. Ser. 373, The Central Engine of Active Galactic Nuclei, ed. L. C. Ho \& J.-W. Wang (San Francisco, CA: ASP), 711

Ho, P. T. P., Moran, J. M., \& Lo, K. Y. 2004, ApJ, 616, L1

Horesh, A., Stockdale, C., Frail, D. A., et al. 2011a, ATel, 3411, 1

Horesh, A., Zauderer, A., \& Carpenter, J. 2011b, ATel, 3405, 1

Hurley, K., Golenetskii, S., Aptekar, R., et al. 2010, in AIP Conf. Ser. 1279, Deciphering the Ancient Universe with Gamma-ray Bursts, ed. N. Kawai \& S. Nagataki (Melville, NY: AIP), 330

Kalberla, P. M. W., Burton, W. B., Hartmann, D., et al. 2005, A\&A, 440, 775

Katz, B., Budnik, R., \& Waxman, E. 2010, ApJ, 716, 781

Katz, B., Sapir, N., \& Waxman, E. 2012, ApJ, 747, 147

Krauss, M. I., Soderberg, A. M., Chomiuk, L., et al. 2012, ApJ, in press (arXiv:1201.0770)

Law, N. M., Kulkarni, S. R., Dekany, R. G., et al. 2009, PASP, 121, 1395

Maddox, L. A., Cowan, J. J., Kilgard, R. E., Schinnerer, E., \& Stockdale, C. J. 2007, AJ, 133, 2559

Margutti, R., \& Soderberg, A. 2011, ATel, 3400, 1

Marion, G. H., Kirshner, R., Wheeler, J. C., et al. 2011, ATel, 3435, 1

Marti-Vidal, I., Tudose, V., Paragi, Z., et al. 2011, A\&A, 535, L10

Matsuoka, M., Kawasaki, K., Ueno, S., et al. 2009, PASJ, 61, 999

Matzner, C. D., \& McKee, C. F. 1999, ApJ, 510, 379

Maund, J. R., Fraser, M., Ergon, M., et al. 2011, ApJ, 739, L37

Mazzali, P. A., Deng, J., Nomoto, K., et al. 2006, Nature, 442, 1018

Mihara, T., Nakajima, M., Sugizaki, M., et al. 2011, PASJ, 63, 623

Murphy, J. W., Jennings, Z. G., Williams, B., et al. 2011, ApJ, 742, L4

Nakar, E., \& Sari, R. 2010, ApJ, 725, 904

Ott, C. D. 2009, Class. Quantum Gravity, 26, 063001

Patnaude, D. J., Loeb, A., \& Jones, C. 2011, New Astron., 16, 187

Pérez-Torres, M. A., Alberdi, A., Marcaide, J. M., et al. 2005, MNRAS, 360, 1055

Perley, R., Napier, P., Jackson, J., et al. 2009, Proc. IEEE, 97, 1448

Pooley, D. 2011, ATel, 3456, 1

Prieto, J. L., \& Hornoch, K. 2011, ATel, 3433, 1

Prieto, J. L., Szczygiel, D. M., Pojmanski, G., \& Beacom, J. F. 2011, ATel, 3432,1

Roming, P. W. A., Pritchard, T. A., Brown, P. J., et al. 2009, ApJ, 704, L118

Ryder, S. D., Sadler, E. M., Subrahmanyan, R., et al. 2004, MNRAS, 349, 1093

Sapir, N., Katz, B., \& Waxman, E. 2011, ApJ, 742, 36 
Sault, R. J., Teuben, P. J., \& Wright, M. C. H. 1995, in ASP Conf. Ser. 77 , Astronomical Data Analysis Software and Systems IV, ed. R. A. Shaw, H. E. Payne, \& J. J. E. Hayes (San Francisco, CA: ASP), 433

Schlegel, E. M., \& Ryder, S. 2002, IAU Circ., 7913, 1

Silverman, J. M., Filippenko, A. V., \& Cenko, S. B. 2011, ATel, 3398, 1

Smartt, S. J. 2009, ARA\&A, 47, 63

Soderberg, A., Grindlay, J. E., Bloom, J. S., et al. 2009, Astro2010: The Astronomy and Astrophysics Decadal Survey, Science White Papers, 278
Soderberg, A. M., Berger, E., Page, K. L., et al. 2008, Nature, 453, 469

Soderberg, A. M., Chevalier, R. A., Kulkarni, S. R., \& Frail, D. A. 2006a, ApJ, 651,1005

Soderberg, A. M., Kulkarni, S. R., Nakar, E., et al. 2006b, Nature, 442, 1014

Van Dyk, S. D., Li, W., Cenko, S. B., et al. 2011, ApJ, 741, L28

Vinko, J., Takats, K., Szalai, T., et al. 2012, A\&A, 540, 93

Waxman, E., Mészáros, P., \& Campana, S. 2007, ApJ, 667, 351

Weiler, K. W., Williams, C. L., Panagia, N., et al. 2007, ApJ, 671, 1959 